From the Department of Molecular Oncology, John Wayne Cancer Institute; Department of Surgical Pathology, Saint John's Health Center, Santa Monica, CA; Department of Surgery, Department of Medical Statistics and Bioinformatics, Leiden University Medical Center, Leiden; and Department of Pathology, Radboud University Nijmegen Medical Center, Nijmegen, the Netherlands.

Submitted August 17, 2007; accepted January 22, 2008.

Supported by the Ruth and Martin $\mathrm{H}$. Weil Fund, the Gonda Foundation, and Coates Laboratories.

Authors' disclosures of potential conflicts of interest and author contributions are found at the end of this article.

Corresponding author: Dave S.B. Hoon, PhD, Department of Molecular Oncology, John Wayne Cancer Institute, 2200 Santa Monica Blvd, Santa Monica, CA 90404; e-mail: hoon@jwci.org.

( 2008 by American Society of Clinica Oncology

0732-183X/08/2614-2327/\$20.00 DOI: 10.1200/JCO.2007.14.0723

\title{
Quantitative Analysis of Methylation of Genomic Loci in Early-Stage Rectal Cancer Predicts Distant Recurrence
}

Michiel F.G. de Maat, Cornelis J.H. van de Velde, Martijn P.J. van der Werff, Hein Putter, Naoyuki Umetani, Elma Meershoek Klein-Kranenbarg, Roderick R. Turner, J. Han J.M. van Krieken, Anton Bilchik, Rob A.E.M. Tollenaar, and Dave S.B. Hoon

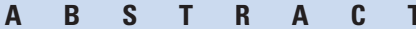

\section{Purpose}

There are no accurate prognostic biomarkers specific for rectal cancer. Epigenetic aberrations, in the form of DNA methylation, accumulate early during rectal tumor formation. In a preliminary study, we investigated absolute quantitative methylation changes associated with tumor progression of rectal tissue at multiple genomic methylated-in-tumor (MINT) loci sequences. We then explored in a different clinical patient group whether these epigenetic changes could be correlated with clinical outcome.

\section{Patients and Methods}

Absolute quantitative assessment of methylated alleles was used to assay methylation changes at MINT 1, 2, 3, 12, 17, 25, and 31 in sets of normal, adenomatous, and malignant tissues from 46 patients with rectal cancer. Methylation levels of these biomarkers were then assessed in operative specimens of 251 patients who underwent total mesorectal excision (TME) without neoadjuvant radiotherapy in a multicenter clinical trial.

\section{Results}

Methylation at MINT 2, 3, and 31 increased 11-fold $(P=.005)$, 15-fold $(P<.001)$, and two-fold $(P=.02)$, respectively, during adenomatous transformation in normal rectal epithelium. Unsupervised grouping analyses of quantitative MINT methylation data of TME trial patients demonstrated two prognostic subclasses. In multivariate analysis of node-negative patients, this subclassification was the only predictor for distant recurrence (hazard ratio $[\mathrm{HR}], 4.17 ; 95 \% \mathrm{Cl}, 1.72$ to 10.10 ; $P=.002)$, cancer-specific survival (HR, 3.74; $95 \% \mathrm{Cl}, 1.4$ to $9.43 ; P=.003)$, and overall survival (HR, 2.68; $95 \% \mathrm{Cl}, 1.41$ to $5.11 ; P=.005)$

\section{Conclusion}

Methylation levels of specific MINT loci can be used as prognostic variables in patients with American Joint Committee on Cancer stage I and II rectal cancer. Quantitative epigenetic classification of rectal cancer merits evaluation as a stratification factor for adjuvant treatment in early disease.

\section{J Clin Oncol 26:2327-2335. (c) 2008 by American Society of Clinical Oncology}

\section{INTRODUCTION}

Rectal cancer is the second most common cancer of the digestive system in the United States. ${ }^{1}$ Neoadjuvant therapy has improved local control of rectal cancer in patients undergoing total mesorectal excision (TME), ${ }^{2-4}$ but distant recurrence remains the major cause of disease mortality. Although tumor status of regional nodes is the most important predictor of metastasis, $20 \%$ of node-negative patients will have a recurrence at distant sites. This suggests that even early stages of tumors have potential for systemic metastasis and, therefore, molecular subclassification may be clinically relevant. Development of prognostic molecular biomarkers for rectal cancer would improve management and potential treatment stratification. Colon and rectal cancers are often assessed together in the analysis of molecular/genetic biomarkers. This is often due to the limited availability of rectal tumor for analysis, or rectal specimens are not procured from a specific clinical trial. We now know both colon and rectal cancers are different in etiology and treatment, as well as (epi)genetics. ${ }^{5}$ In this study, we have focused specifically on epigenetic changes of rectal cancers from a clinical trial.

Epigenetic instability, such as changes in genomic DNA methylation status, is an early event during GI tumor development and encompasses both hyper- and hypomethylation changes. ${ }^{6-8}$ Most epigenetic cancer studies focus on specific genomic 
loci and analyze methylation status in a dichotomous manner, categorizing specimens as methylated or unmethylated. Also, in the majority of the studies assessing epigenetic changes and association with clinical outcome, nonquantitative measures are used, using a binary methylation status result. Absolute quantitative interpretation of methylation data would improve analysis of epigenetic events. ${ }^{9} \mathrm{Re}-$ cently, we developed an assay for absolute quantitative assessment of methylated alleles (AQAMA) and showed quantitative methylation events to be associated with colorectal tumor progression. ${ }^{10}$ AQAMA measures the amount of methylated and unmethylated copy numbers simultaneously in a single reaction. The assay has excellent linearity in assessing DNA methylation levels and can be used on paraffinembedded archival tissue (PEAT) sections treated with the on-slide (in situ) sodium bisulfite modification (SBM) technique that allows microdissected histology-oriented assessment of small (1 to $2 \mathrm{~mm}^{2}$ ) lesions. ${ }^{11,12}$ This allows efficient comparison of precursor adenoma and normal cells adjacent to tumor cells.

Methylation levels of methylated-in-tumor (MINT) loci have not been specifically tested for prognostic utility in rectal cancer. MINT loci are CpG dinucleotide-rich regions located in nonproteinencoding DNA regions, and have been reported to become methylated in a tumor- and adenoma-specific manner in gastric and colon cancer. ${ }^{13-17}$ In a preliminary study, we quantified methylation levels of seven MINT loci at different stages of rectal tumor formation comparing paired normal-adenoma and adenoma-cancer tissues, and subsequently analyzed whether methylation level changes related to rectal tumor progression. Our developed hypothesis was that methylation levels at MINT loci have prognostic significance for early rectal cancer progression. We then assessed the potential prognostic utility of MINT loci in primary tumor tissues from patients enrolled in a multicenter, randomized, surgical clinical trial. In this translational study analysis, unsupervised cluster analysis identified a subclass of patients whose quantitative methylation data were independently prognostic of progression to distant disease.

\section{PATIENTS AND METHODS}

\section{Tissue Specimens}

In the preliminary study, patients who underwent surgery for rectal cancer with histopathologic-confirmed adenocarcinoma were identified from the cancer registry database at Saint John's Health Center (Santa Monica, CA). Only patients who underwent surgery after 1995 were evaluated because of possible DNA degradation. Further selection of specimens was based on pathology-documented presence of tumor, as well as adenoma cells on the same tissue section.

For the clinical correlation studies, primary tumor PEAT specimens were obtained from 322 nonirradiated patients enrolled onto the multicenter, randomized, quality-controlled TME trial coordinated by the Dutch Colorectal Cancer Group. ${ }^{3}$ The trial investigated whether neoadjuvant radiotherapy $(5 \times 5$ Gy) before TME improved local control compared with TME surgery alone in patients with all stages of rectal cancer. Trial eligibility criteria and follow-up protocols have been described previously. ${ }^{3,18,19}$ All TME trial patients enrolled at the Dutch multicenter study sites were eligible, further adhering to the following criteria: nonirradiated, TNM stage I-III, with no evidence of disease after surgery. We opted to analyze the nontreatment arm because potential effects of radiation on genomic methylation are not known. Research protocols for the methylation studies on PEAT were approved by the internal review boards of Saint John's Health Center, John Wayne Cancer Institute (Santa Monica, CA), and Leiden University Medical Center (Leiden, the Netherlands).

\section{DNA Preparation From PEAT Specimens for Preliminary and Clinical Studies}

From the preliminary study specimens, two consecutive sections ( 4 and $7 \mu \mathrm{m}$ ) of each PEAT block were cut and placed on adhesive-coated slides. The $4 \mu \mathrm{m}$ section was stained with hematoxylin and eosin (H\&E) and mounted. Tissue areas with normal epithelial, classic adenomatous, and invasive cancer cells were identified and marked by an expert surgical pathologist (R.R.T.). The tissue categories were identified by histopathology. Cancer cells were only taken from areas with nuclear atypia and signs of invasion of tissue architectural boundaries, the hallmark of cancer. Adenomatous cells were only taken from areas with classic villous and/or tubular adenomatous dysplasia. We did not include adenomatous tissue in the analysis with highly dysplastic features without signs of invasion. The 7- $\mu \mathrm{m}$ section was treated by on-slide SBM as described previously. ${ }^{11}$ Target tissue areas were identified and microdissected under a light microscope. Isolated cells were digested and $1 \mu \mathrm{L}$ of the lysate was used for polymerase chain reaction.

From the clinical study of TME trial patient specimens, tissue sections (7 $\mu \mathrm{m})$ were cut from PEAT specimens and mounted on nonadhesive glass slides. Tumor-representative areas on H\&E-stained sections were marked by a surgical pathologist specializing in rectal cancer (J.H.J.M.vK.). Two sections per patient were deparaffinized, and the marked tissue was carefully microdissected. DNA was isolated and modified by sodium bisulfite, as previously described. ${ }^{20}$ Salmon sperm DNA was added as a carrier. ${ }^{21}$ Double-stranded DNA and single-stranded DNA were quantified before and after SBM by PicoGreen and OliGreen assays (Molecular Probes; Invitrogen, Carlsbad, CA), respectively. Sufficient input DNA for AQAMA was determined as described. ${ }^{10}$ A salmon sperm DNA sample without tumor DNA was included in triplicate to assess background signal in all assays. Tissue blocks and isolated DNA were coded to prevent any bias.

\section{AQAMA MINT Locus Methylation Level Assessment}

Absolute quantitative assessment of methylated alleles at MINT loci 1,2, 12 , and 31 has been described previously. ${ }^{10}$ Unpublished primer and probe sets for the remaining three MINT loci were: MINT3, 5'-TGATGGTGTAT GTGATTTTGTGTT-3' (forward), 5' -ACCCCACCCCTCACAAAC-3' (reverse), 5'-ACCTACGAACGAACAC-3' (methylated probe), 5'-TACCTACAAAC AAACAC-3' (unmethylated probe); MINT17, 5'-AGGGGTTAGGTTG AGGTTGTT-3' (forward), 5' -TCTACCTCTTCCCAAATTCCA-3' (reverse), 5' TTGGATGGATCGCGG-3' (methylated probe), 5'-TATTTTGGATGGA TTGTGG-3' (unmethylated probe); and MINT25, 5'-GGGGATAGGAAGA TGGTTT-3' (forward), 5'-CCCCCATCCCATACAACC-3' (reverse), 5'-TTT GTTTCGTAGCGGAGT-3'(methylated probe), 5'-GATTTTGTTTTGTAGT GGAG-3' (unmethylated probe). DNA samples were run in 384-well microplates in triplicate, and each plate contained individual marker cDNA standards with known copy numbers, allowing assessment of absolute methylated and unmethylated copy number. Controls for specificity of AQAMA for methylated and unmethylated sequences, as well as controls for nonspecific amplification, were included. ${ }^{10,22}$ Final analysis outcome was the methylation index (MI), calculated as: [copy number methylated alleles (copy number ${ }_{\text {methylated alleles }}$ + copy number $\left.{ }_{\text {unmethylated alleles }}\right)$.

\section{Profiling by Unsupervised Random Forest Clustering}

For identification of patient clusters with similar MINT locus methylation profiles, we employed unsupervised random forest (RF) clustering. ${ }^{23} \mathrm{RF}$ has been successfully applied in comparable data sets (Appendix 1, online only). ${ }^{24,25}$

\section{RESULTS}

\section{MINT Locus Methylation Levels During Rectal Cancer Development}

Sets of normal, adenomatous, and malignant PEAT tissues from 46 patients with rectal cancer were examined by AQAMA of MINT loci known to be differentially methylated in colorectal cancer. ${ }^{12}$ The $\mathrm{H} \& \mathrm{E}$-stained sections cut from the tissue blocks that, according to the 


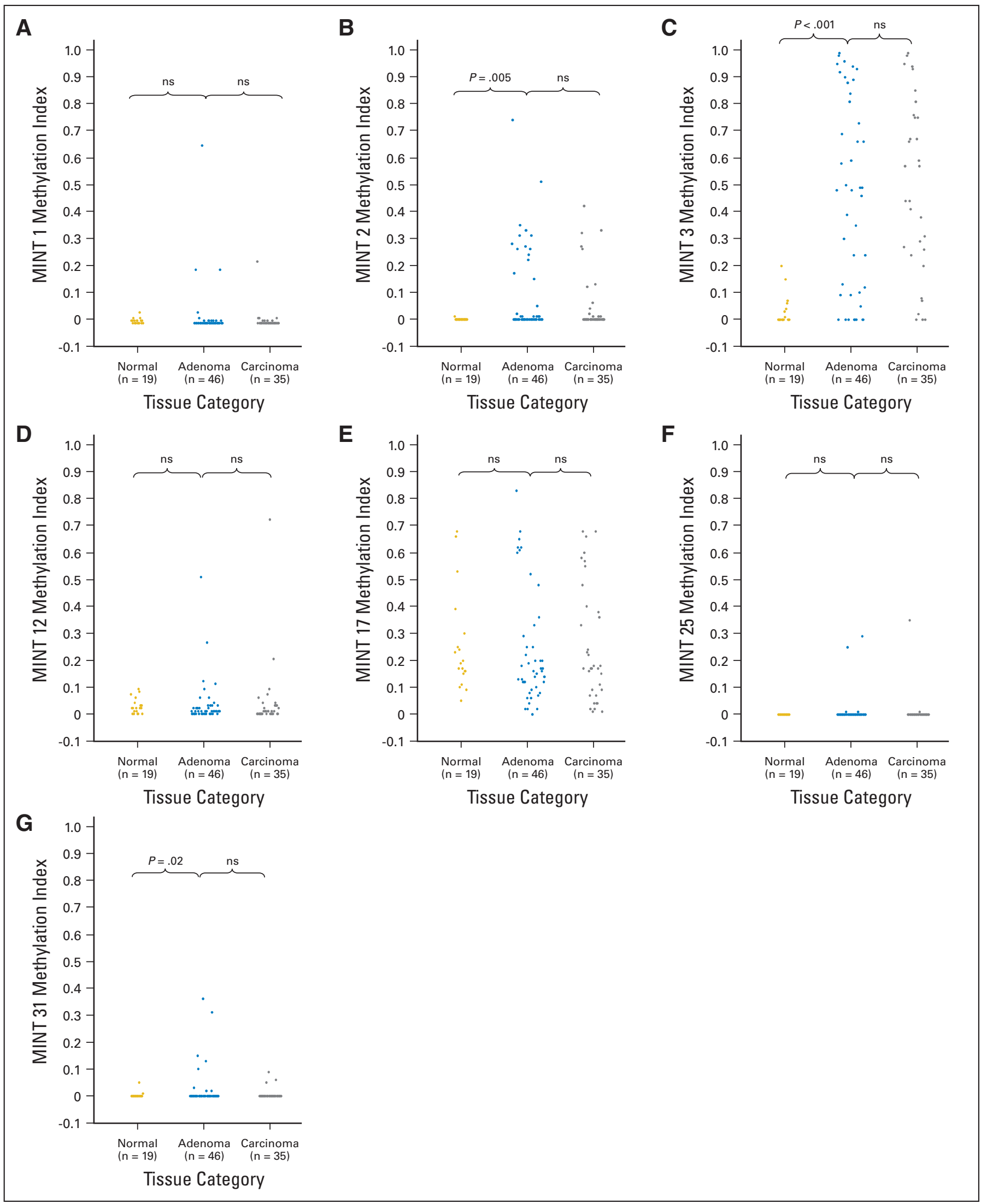

Fig 1. Scatterplots of measured methylation indices in normal rectal epithelium, rectal adenoma tissue, and rectal cancer tissue for the 7 MINT loci studied. MINT, methylated in tumor; ns, not significant. 


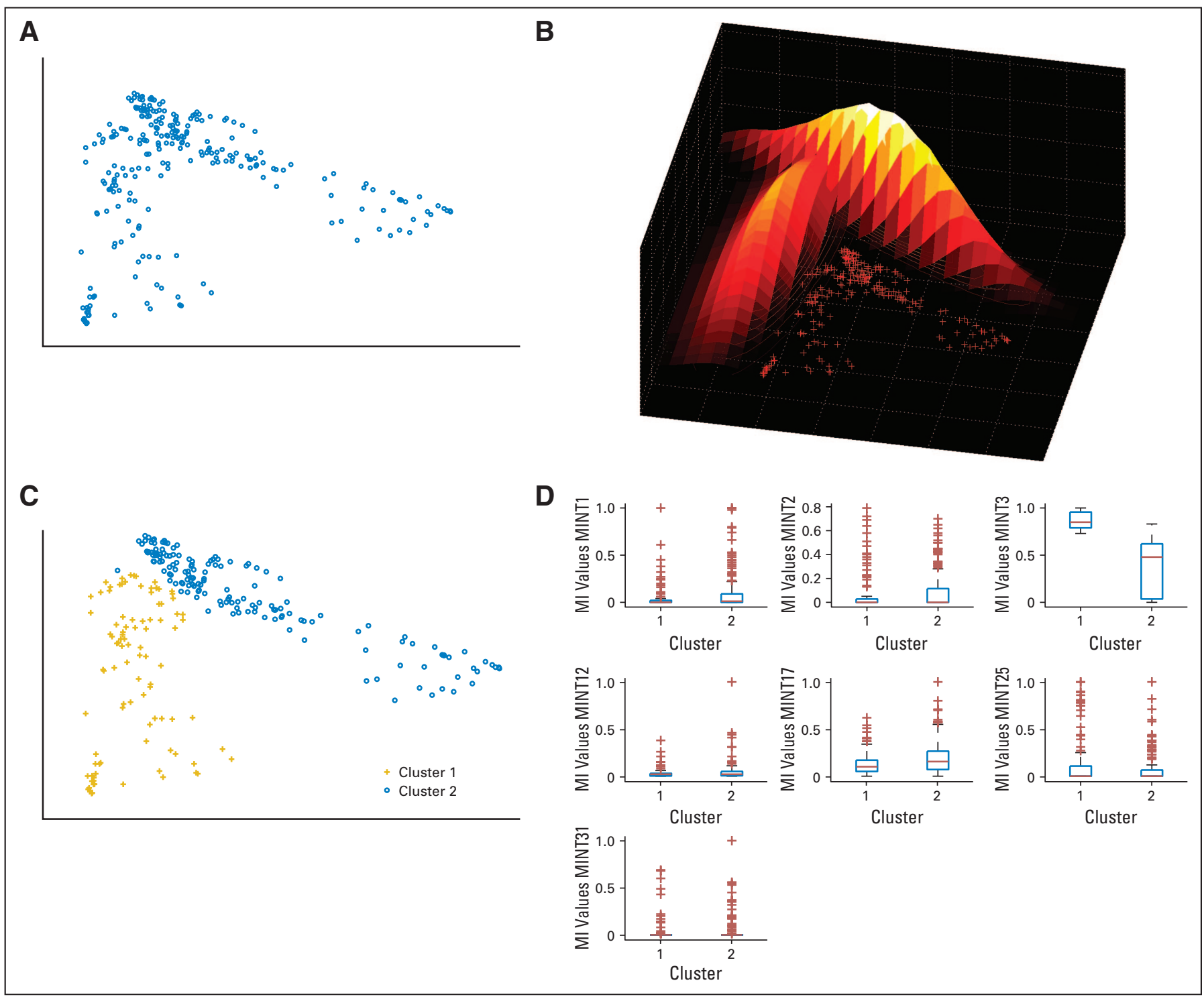

Fig 2. Methylated in tumor (MINT) locus methylation subclass identification. (A) multidimensional scaling [MDS] plot displaying the level of dissimilarity between all patients (MDS plot axes represent arbitrary units, and are therefore dimensionless). (B) Three-dimensional plot representing expectation maximization algorithm with a mixture of Gaussians analysis of the MDS plot coordinates showing Gaussian distribution (bell-shaped) of the two identified clusters. (C) MDS plot showing final cluster allocations for the patient population. (D) Box plots comparing the differences in methylation levels (MI) between cluster 1 and 2 for all MINT loci.

diagnostic pathology report, contained adenoma as well as cancer tissue, were evaluated histopathologically by an expert pathologist (R.R.T). In the 46 tissue sections, 19, 46, and 35 areas of normal epithelium, adenoma, and cancer tissue, respectively, were identified. This resulted into paired analyses of 19 normal-adenoma sets and 35 adenoma-cancer sets. Figure 1 shows scatterplots of the MI values in the three histopathology categories for each MINT locus. MINT loci 2, 3 , and 31 underwent a significant increase in absolute mean methylation level during adenomatous transformation. There were no significant MINT methylation changes for any MINT locus during progression from adenoma to cancer. Subsequently, the significant increases were early events associated with dysplastic change of normal rectal epithelium. Because three MINT loci (2, 3, and 31) showed significant increase in methylation levels and the normal distribution of the quantitative methylation data sets in healthy rectal epithelium changes to non-normal in adenoma in four other loci $(1,12,17$, and 25; Appendix 2 and Table A1, online only), all seven MINT loci were considered to have potential utility to identify epigenetic subclasses in the clinical study patient group.

\section{Sample Size Calculations}

To establish the sample size for the clinical study, we performed power calculations using methylation results of the preliminary study and recurrence rates of the TME trial. It was calculated that 250 patients were sufficient to obtain significance for predicting distant recurrence with an $\alpha$ level of $.05 \%$ and $90 \%$ power. Because the available patient specimens from the trial were primary tumor PEAT blocks from various hospital sites, we allowed for $30 \%$ loss of patient 


\begin{tabular}{|c|c|c|c|c|c|c|c|c|c|c|c|}
\hline \multirow[b]{3}{*}{$\begin{array}{l}\text { MINT } \\
\text { Locus }\end{array}$} & \multirow[b]{3}{*}{$\begin{array}{l}\text { Gini } \\
\text { Index }\end{array}$} & \multicolumn{5}{|c|}{ All Patients $(n=251)$} & \multicolumn{5}{|c|}{ Node-Negative Patients $(n=145$ ) } \\
\hline & & \multicolumn{2}{|c|}{ Cluster 1 ( $n=89$ ) } & \multicolumn{2}{|c|}{ Cluster $2(n=162)$} & \multirow[b]{2}{*}{$P^{*}$} & \multicolumn{2}{|c|}{ Cluster $1(n=55)$} & \multicolumn{2}{|c|}{ Cluster 2 (n = 90) } & \multirow[b]{2}{*}{$P^{*}$} \\
\hline & & Median & $\begin{array}{l}\text { Interquartile } \\
\text { Range }\end{array}$ & Median & $\begin{array}{l}\text { Interquartile } \\
\text { Range }\end{array}$ & & Median & $\begin{array}{l}\text { Interquartile } \\
\text { Range }\end{array}$ & Median & $\begin{array}{l}\text { Interquartile } \\
\text { Range }\end{array}$ & \\
\hline MINT1 & 11.6 & 0.00 & $0.00-0.01$ & 0.01 & $0.00-0.09$ & $<.001$ & 0.00 & $0.00-0.02$ & 0.00 & $0.00-0.09$ & .006 \\
\hline MINT2 & 10.8 & 0.08 & $0.00-0.02$ & 0.00 & $0.00-0.12$ & .07 & 0.00 & $0.00-0.03$ & 0.00 & $0.00-0.10$ & .51 \\
\hline MINT3 & 20.2 & 0.87 & $0.79-0.99$ & 0.50 & $0.06-0.65$ & $<.001$ & 0.84 & $0.79-0.99$ & 0.49 & $0.06-0.65$ & $<.001$ \\
\hline MINT12 & 13.5 & 0.03 & $0.00-0.02$ & 0.02 & $0.01-0.05$ & .01 & 0.02 & $0.00-0.02$ & 0.02 & $0.00-0.05$ & .22 \\
\hline MINT17 & 20.7 & 0.08 & $0.04-0.13$ & 0.21 & $0.08-0.30$ & $<.001$ & 0.09 & $0.05-0.15$ & 0.20 & $0.12-0.24$ & .005 \\
\hline MINT25 & 12.1 & 0.00 & $0.00-0.04$ & 0.00 & $0.00-0.08$ & .21 & 0.00 & $0.00-0.05$ & 0.00 & $0.00-0.09$ & .81 \\
\hline MINT31 & 6.0 & 0.00 & $0.00-0.00$ & 0.00 & $0.00-0.00$ & .90 & 0.00 & $0.00-0.00$ & 0.00 & $0.00-0.00$ & .82 \\
\hline
\end{tabular}

samples due to availability and quality of tissue and DNA. We therefore required 75 additional patient samples, and the final sample size was set at 325 patients samples. Six hundred seventy-two patients fulfilled our study criteria (see Patients and Methods). Finally, DNA was isolated in 314 patient samples (in 11 patient samples, tumor cell number was insufficient). Subsequently, after processing and bisulfite treatment, only 251 of the 314 DNA isolations had sufficient input DNA for AQAMA. Characteristics of the 251 patients in the final analysis were not significantly different in prognostic factors and characteristics from the original trial population (Appendix Table A2, online only).

\begin{tabular}{|c|c|c|c|c|c|c|c|c|c|c|}
\hline \multirow[b]{3}{*}{$\begin{array}{c}\text { Clinical and Tumor Pathology } \\
\text { Factors }\end{array}$} & \multicolumn{4}{|c|}{ All Patients $(n=251)$} & \multirow[b]{3}{*}{$P$} & \multicolumn{4}{|c|}{ Node-Negative Patients (145) } & \multirow[b]{3}{*}{$P$} \\
\hline & \multicolumn{2}{|c|}{$\begin{array}{l}\text { Cluster } 1 \\
(\mathrm{n}=89) \\
\end{array}$} & \multicolumn{2}{|c|}{$\begin{array}{l}\text { Cluster } 2 \\
(\mathrm{n}=162) \\
\end{array}$} & & \multicolumn{2}{|c|}{$\begin{array}{l}\text { Cluster } 1 \\
(n=55)\end{array}$} & \multicolumn{2}{|c|}{$\begin{array}{l}\text { Cluster } 2 \\
(\mathrm{n}=90)\end{array}$} & \\
\hline & $\begin{array}{l}\text { No. of } \\
\text { Patients }\end{array}$ & $\%$ & $\begin{array}{l}\text { No. of } \\
\text { Patients }\end{array}$ & $\%$ & & $\begin{array}{l}\text { No. of } \\
\text { Patients }\end{array}$ & $\%$ & $\begin{array}{l}\text { No. of } \\
\text { Patients }\end{array}$ & $\%$ & \\
\hline \multicolumn{11}{|l|}{ Sex } \\
\hline Male & 64 & 39 & 98 & 61 & .08 & 38 & 40 & 56 & 60 & .40 \\
\hline Female & 25 & 28 & 64 & 72 & & 17 & 33 & 34 & 67 & \\
\hline \multicolumn{11}{|l|}{ Age, years } \\
\hline Mean & \multicolumn{2}{|c|}{64.8} & \multicolumn{2}{|c|}{62.5} & .15 & \multicolumn{2}{|c|}{65.4} & \multicolumn{2}{|c|}{63.4} & .33 \\
\hline SE & \multicolumn{2}{|c|}{1.2} & \multicolumn{2}{|c|}{0.9} & & \multicolumn{2}{|c|}{1.7} & \multicolumn{2}{|c|}{1.2} & \\
\hline \multicolumn{11}{|l|}{ TNM stage } \\
\hline 1 & 29 & 41 & 41 & 59 & .22 & 29 & 41 & 41 & 59 & .40 \\
\hline ॥ & 26 & 35 & 49 & 65 & & 26 & 35 & 49 & 65 & \\
\hline III & 34 & 32 & 72 & 68 & & - & & - & & \\
\hline \multicolumn{11}{|l|}{$\mathrm{N}$ status } \\
\hline No ( $\geq 12$ examined) & 12 & 35 & 22 & 65 & .53 & 12 & 35 & 22 & 65 & .84 \\
\hline No/NX (< 12 examined) & 44 & 39 & 68 & 61 & & 43 & 39 & 68 & 61 & \\
\hline N1 (1-3 positive) & 21 & 34 & 40 & 66 & & - & & - & & \\
\hline N2 ( $\geq 4$ positive) & 12 & 27 & 32 & 73 & & - & & - & & \\
\hline \multicolumn{11}{|l|}{ Differentiation } \\
\hline Well & 5 & 28 & 13 & 72 & .78 & 3 & 33 & 6 & 67 & .99 \\
\hline Moderately & 66 & 37 & 110 & 63 & & 44 & 39 & 70 & 61 & \\
\hline Poor & 18 & 32 & 39 & 68 & & 8 & 36 & 14 & 64 & \\
\hline \multicolumn{11}{|l|}{ Location of distant recurrences } \\
\hline Liver & 11 & 41 & 16 & 59 & .62 & 4 & 50 & 4 & 50 & .37 \\
\hline Not liver & 20 & 49 & 21 & 51 & & 11 & 73 & 4 & 27 & \\
\hline \multicolumn{11}{|l|}{ Resection type } \\
\hline Low anterior & 54 & 32 & 113 & 68 & .26 & 32 & 34 & 62 & 66 & .35 \\
\hline Abdominoperineal & 33 & 43 & 44 & 57 & & 21 & 47 & 24 & 53 & \\
\hline Hartmann & 2 & 29 & 5 & 71 & & 2 & 33 & 4 & 67 & \\
\hline \multicolumn{11}{|l|}{ Circumferential margin } \\
\hline Negative & 72 & 35 & 131 & 65 & .39 & 52 & 39 & 81 & 61 & .54 \\
\hline Positive & 17 & 35 & 31 & 65 & & 3 & 25 & 9 & 75 & \\
\hline
\end{tabular}




\section{MINT Locus Methylation Profile Identification}

To investigate whether rectal cancer can be grouped by methylation level at specific MINT loci, we performed unsupervised RF clustering on the quantitative methylation level results of patients from the TME trial. As an outcome, a multidimensional scaling (MDS) plot indicated the mutual distance between the samples based on methylation level of all seven MINT loci (Fig 2A). Inspection of the MDS plot indicated two groups of rectal cancer cases. To identify which patients belonged to which group, we performed an expectation maximization algorithm with a mixture of Gaussians (EM-MoG) analysis based on the Gaussian shape of patient clusters (Figs 2B and 2C). The EM-MoG algorithm allocated the patients based on the likelihood that they would fall under the normal (Gaussian) distribution of one of the two clusters. Subsequently, variable importance and the methylation patterns matching the identified clusters were analyzed (Fig 2D; Table 1). The 89 patients (35\%) allocated to cluster 1 had significantly increased methylation at MINT3 and significantly decreased methylation at MINT1, 12, and 17 compared with patients in cluster 2 . The unsupervised clustering results showed that subclasses of rectal cancers could be identified by differences in DNA methylation level of tested MINT loci. The Gini index indicated that MINT3 and MINT17 were the most important variables in forming the clusters.

\section{Clinicopathologic Correlation and Distant Recurrence Analyses}

There were no significant associations observed in epigenetic subclasses of rectal cancer to any of the investigated standard clinical or tumor-pathological factors (Table 2). The preliminary results demonstrated that methylation level differences at the specific MINT loci develop early during tumor formation. There was no significant relation between cluster allocation and clinicopathologic factors in nodenegative tumors (Table 2). Because identification of stage I and II patients at risk for distant metastasis is clinically highly relevant and there was no dependence of the identified patient clusters to nodal status, we excluded stage III patients from distant disease recurrence analyses. We assessed the probability of distant disease recurrence, cancer-specific, and overall survival (OS). Because EM-MoG analysis is a probability-based cluster assignment algorithm, we performed multiple imputation analysis to correct for cases that have a small difference in probability to be assigned to either one of the clusters. In node-negative patients, cluster 1 patients had significant increased risk for distant recurrence $(P=.01)$, shorter cancer-specific survival $(P=.02)$, and shorter OS $(P=.05$; Figs $3 \mathrm{~A}$ to $3 \mathrm{C})$. At the time of the analyses, median duration of follow-up was 7.1 years (range, 2.5 to 9.8 years).

\section{Multivariate Analyses}

Multivariate analyses were performed to assess whether the observed prognostic value of the clusters was independent from standard prognostic variables for the complete patient group and for nodepositive and negative patients (Table 3 ). $\mathrm{T}$ stage, $\mathrm{N}$ stage, circumferential margin status, distance of the tumor to the anal verge, and tumor differentiation were considered in a Cox regression analysis. In node-negative patients, the quantitative MINT locus methylation profile — of all the considered variables — was the only selected predictive factor for distant disease recurrence and cancer-specific survival. OS was also affected by T-stage in patients without nodal involvement.

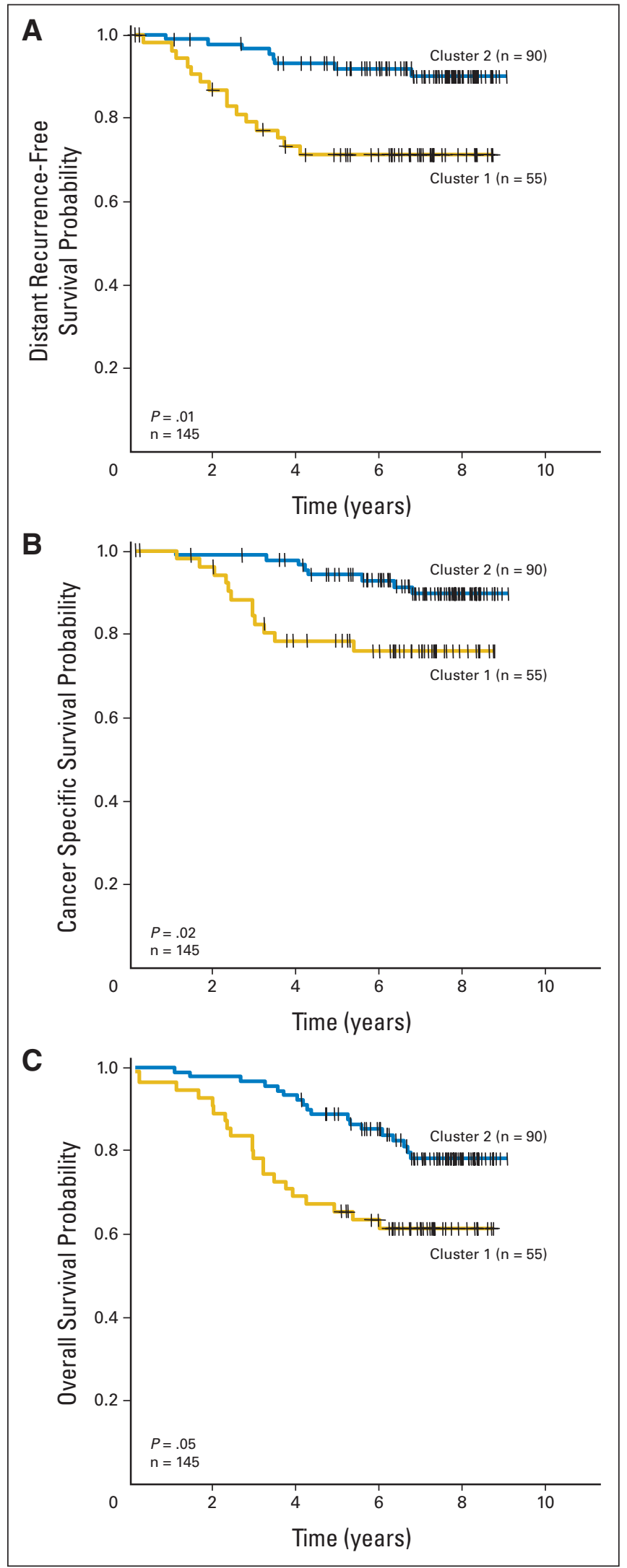

Fig 3. (A) Kaplan-Meier plots grouping analyzed node-negative total mesorectal excision trial patients into clusters 1 and 2 and comparing postoperative distant recurrence free survival probability. (B) Cancer-specific and (C) overall survival are plotted. 


\begin{tabular}{|c|c|c|c|c|c|c|c|c|c|}
\hline \multirow[b]{2}{*}{ Variable } & \multicolumn{3}{|c|}{$\begin{array}{l}\text { All Patients } \\
(n=251)\end{array}$} & \multicolumn{3}{|c|}{$\begin{array}{l}\text { Node Negative } \\
\quad(n=145)\end{array}$} & \multicolumn{3}{|c|}{$\begin{array}{l}\text { Node Positive } \\
\quad(n=106)\end{array}$} \\
\hline & $\mathrm{HR}$ & $95 \% \mathrm{Cl}$ & $P$ & $\mathrm{HR}$ & $95 \% \mathrm{Cl}$ & $P$ & $\mathrm{HR}$ & $95 \% \mathrm{Cl}$ & $P$ \\
\hline \multicolumn{10}{|l|}{ Distant recurrence } \\
\hline T stage (3/4) & 1.70 & 0.92 to 3.16 & .09 & 1.19 & 0.49 to 2.93 & .70 & 2.91 & 1.01 to 8.37 & .05 \\
\hline Nodal status $(+)$ & 2.47 & 1.44 to 4.23 & .001 & - & & - & - & & NS \\
\hline Circumferential margin $(+)$ & 1.87 & 1.07 to 3.28 & .03 & 2.40 & 0.62 to 9.39 & .21 & 1.77 & 0.94 to 3.33 & .08 \\
\hline Distance from anal verge $>5 \mathrm{~cm}$ & 0.71 & 0.43 to 1.18 & .19 & 1.50 & 0.59 to 3.85 & .40 & 0.50 & 0.27 to 0.92 & .03 \\
\hline Poor differentiation & 1.39 & 0.81 to 2.38 & .23 & 1.24 & 0.40 to 3.89 & .71 & 1.59 & 0.84 to 3.01 & .16 \\
\hline MINT locus profile (cluster 1) & 1.68 & 1.03 to 2.73 & .04 & 4.17 & 1.72 to 10.10 & .002 & 1.11 & 0.59 to 2.09 & .75 \\
\hline \multicolumn{10}{|l|}{ Cancer-specific survival } \\
\hline T-stage (3/4) & 2.12 & 1.07 to 4.19 & .03 & 1.88 & 0.70 to 5.04 & .21 & 2.85 & 0.98 to 8.26 & .05 \\
\hline Nodal status (+) & 2.47 & 1.41 to 4.35 & .002 & - & & - & - & & NS \\
\hline Circumferential margin (+) & 1.93 & 1.09 to 3.41 & .02 & 2.28 & 0.59 to 8.81 & .23 & 1.88 & 0.99 to 3.56 & .05 \\
\hline Distance from anal verge $>5 \mathrm{~cm}$ & 0.59 & 0.35 to 0.99 & .05 & 1.46 & 0.53 to 4.03 & .46 & 0.40 & 0.22 to 0.75 & .004 \\
\hline Poor differentiation & 1.56 & 0.91 to 2.70 & .11 & 1.28 & 0.41 to 4.06 & .67 & 1.70 & 0.88 to 3.29 & .12 \\
\hline MINT locus profile (cluster 1) & 1.47 & 0.88 to 2.45 & .15 & 3.74 & 1.48 to 9.43 & .005 & 0.99 & 0.51 to 1.93 & .98 \\
\hline \multicolumn{10}{|l|}{ Overall survival } \\
\hline T stage $(3 / 4)$ & 1.92 & 1.14 to 3.23 & .01 & 2.12 & 1.05 to 4.29 & .04 & 1.98 & 0.87 to 4.50 & .10 \\
\hline Nodal status (+) & 1.88 & 1.22 to 2.92 & .004 & - & & NS & - & & NS \\
\hline Circumferential margin $(+)$ & 1.66 & 1.02 to 2.69 & .04 & 1.65 & 0.60 to 4.53 & .33 & 1.66 & 0.95 to 2.91 & .08 \\
\hline Distance from anal verge $>5 \mathrm{~cm}$ & 0.69 & 0.45 to 1.06 & .09 & 0.96 & 0.49 to 1.90 & .92 & 0.55 & 0.32 to 0.95 & .03 \\
\hline Poor differentiation & 1.33 & 0.84 to 2.09 & .22 & 1.06 & 0.45 to 2.47 & .90 & 1.37 & 0.77 to 2.44 & .28 \\
\hline MINT locus profile (cluster 1 ) & 1.48 & 0.98 to 2.24 & .06 & 2.68 & 1.41 to 5.11 & .003 & 1.00 & 0.57 to 1.77 & 1.00 \\
\hline
\end{tabular}

Abbreviations: HR, hazard ratio; MINT, methylated in tumor; NS, not significant.

Circumferential margin involvement of the tumor and short $(<5 \mathrm{~cm})$ distance of the tumor from the anal verge increased the risk of distant recurrence, and decreased cancer-specific survival and OS in nodepositive rectal cancer patients. Possible dependence of the results on any of the 42 different study sites was evaluated in the published clinical trial report ${ }^{26}$ and was also ruled out (data not shown) in our analyses. The multivariate results show that the identified subclass of rectal cancers is independently predictive of distant recurrence in node-negative patients.

\section{MINT3 and MINT17}

The Gini index, indicating variable importance in RF clustering as shown in Table 1, demonstrated MINT3 and MINT17 to hold the most information to form the two clusters compared with the other five MINT loci. We continued to assess whether methylation levels at MINT3 and MINT17 have prognostic value as a separate marker set. The quantitative methylation data of MINT3 and MINT17 were entered into the RF algorithm and the resulting MDS plot is shown in Figure 4A. Four clearly separate clusters are formed and the corresponding methylation level differences between the clusters are plotted in Figure 4B. Cluster 3, with 67 patients (27\%), corresponds to the previously identified high-risk cluster 1 because the average MINT3 methylation index is relatively high and the MINT17 methylation index is relatively low. In Kaplan-Meier analysis, cluster 3 patients are shown to be at significantly increased risk for distant metastasis in node-negative patients compared with the other three clusters (Fig 4C). In multivariate analysis, the results showed that the high-risk cluster 3 was selected as the only independent factor among the variables analyzed that in node-negative patients predicted distant recurrence probability (hazard ratio $[\mathrm{HR}], 2.84 ; 95 \% \mathrm{CI}, 1.22$ to
$6.62 ; P=.02)$, cancer-specific survival (HR, 3.29; 95\% CI, 1.33 to $8.12 ; P=.01)$, and OS (HR, $2.21 ; 95 \% \mathrm{CI}, 1.13$ to $4.29 ; P=.02)$. It was concluded that patients at increased risk for distant metastasis can be defined as having tumors with a MINT3 methylation level more than 0.72 and MINT17 methylation level less than 0.14. The analysis also demonstrated that the specific combination of increased methylation at MINT3 and decreased methylation at MINT17 is required for the prognostic information.

\section{DISCUSSION}

Most studies of biomarkers in large bowel adenocarcinoma include both the colon and the rectum, even though rectal and colon cancers are treated differently. Moreover, right-sided and left-sided bowel adenocarcinomas have different molecular patterns; microsatellite instability and methylator phenotype are rarely seen in the rectum. ${ }^{27}$ Our data represent one of the largest clinical analyses of methylation biomarkers in rectal cancer specifically, and to our knowledge also demonstrate the first quantitative correlation between MINT methylation levels and disease progression.

The preliminary study demonstrated a progressive increase in methylation levels of specific MINT loci comparing normal and adenomatous rectal tissue. No significant change in methylation level at any MINT locus was detected comparing adenomatous and malignant rectal tissue. A correlation between methylation of MINT loci and development of adenomatous dysplasia has been reported. ${ }^{17}$ Our data are unique, as we used paired normal-adenoma cancer specimens, quantitative techniques, and analyzed rectal cancers only. The results of our clinical study identified two prognostic categories of 


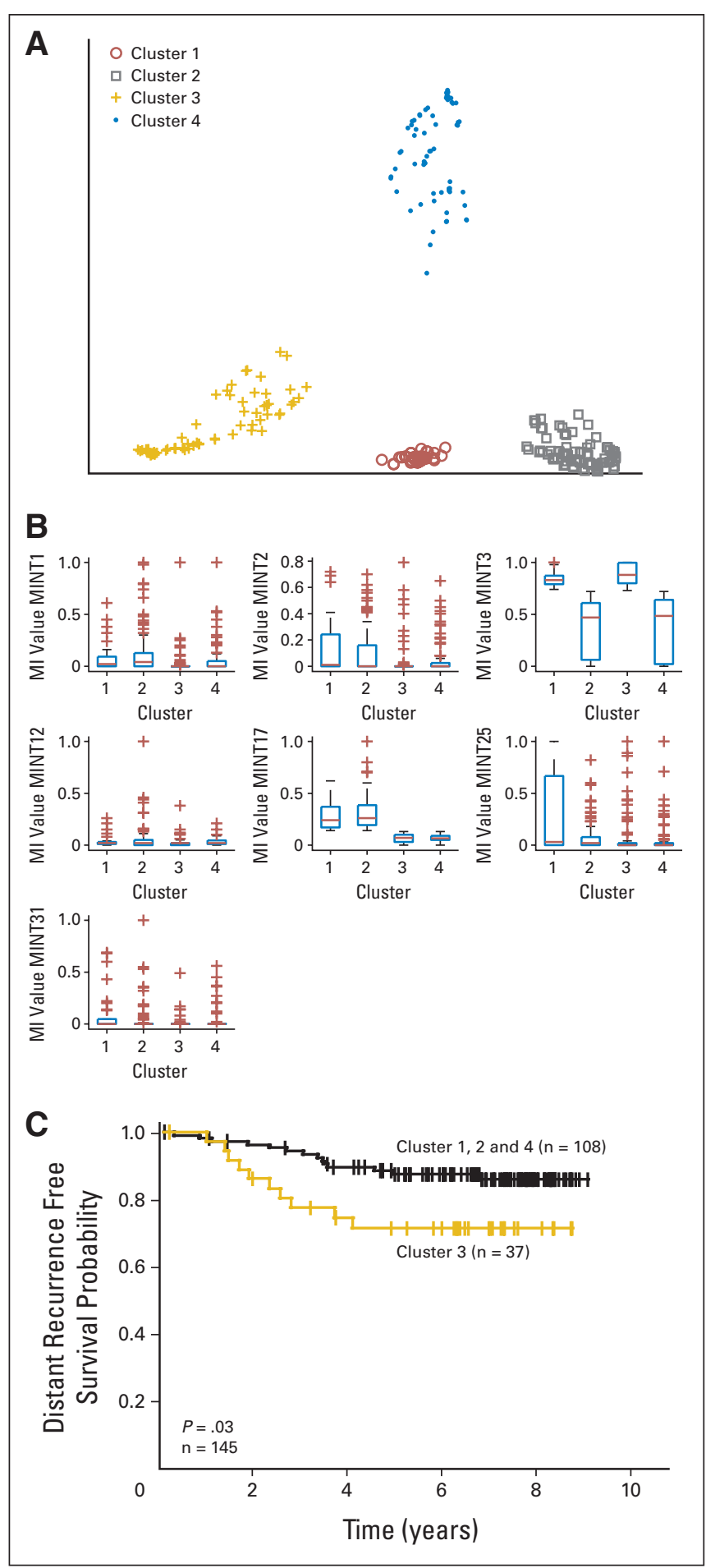

Fig 4. Random forest analyses using only MINT3 and 17 quantitative methylation data as input. (A) Multidimensional scaling plot showing the four clusters. (B) Box plots comparing the differences in methylation levels (MI) between the four clusters. (C) Kaplan-Meier plot illustrates the distant recurrence probability between high-risk cluster 3 and the combined clusters 1, 2, and 4 .

rectal cancer based strictly on the absolute quantitative differences in methylation level. Our data show that methylation levels at multiple and two specific identified MINT loci are related to rectal tumor formation, and that they may be seen as surrogate markers of distant rectal cancer disease recurrence and disease survival. The role of noncoding regions has been of much interest in that it may be influential in gene encoding regions. ${ }^{28-30}$ Especially interesting is that the chromosomal location (1p36) of the MINT3 locus, which undergoes methylation in most rectal adenomas, contains many cancer-related genes. Methylation of MINT loci 1,2, 12, and 31 is often studied in relation to the CpG island methylator phenotype (CIMP) that forms a subclass of right colon tumors closely associated with microsatellite instability. ${ }^{31}$ In our study, the unsupervised clustering analyses did not identify a CIMP associated with hypermethylation in the selected MINT loci (data not shown). Interestingly, a combination of relative hyper- as well as hypomethylation was observed in the identified subclasses. This specific combination was even required to show prognostic value on rectal cancer distant recurrence rates. This corroborates that CIMP does not occur in the rectum and that rectal cancer may have different epigenetic pathologic changes compared with proximal colon adenocarcinoma. Reported correlations between MINT 1, 2, 12, and 31 and clinicopathologic features overlap with the features of microsatellite instability (positive) tumors (right-sidedness, poor differentiation, early stage) and therefore our results cannot be compared. ${ }^{27,32,33} \mathrm{We}$ previously showed relevance of the AQAMA technique testing methylation levels at MINT 1, 2, 12, and 31 and increased methylation at this loci detected by the AQAMA assay was significantly correlated to right-sided colon tumors. ${ }^{10}$

Our preliminary study data indicate that methylation events at the measured MINT loci are related to early dysplastic proliferation of subclasses of rectal premalignancies, and MINT loci may be a clinical biomarker. Subsequently, in a large rectal cancer patient group, RF clustering was able to identify - in an unbiased manner - two groups of rectal cancer patients that were naturally present within the quantitative methylation data. This demonstrated that subclassification of rectal cancer patients can be made based on absolute quantitative methylation level differences.

There was no correlation between MINT methylation profile and nodal status; in node-negative patients, the MINT profile was the only selected variable in multivariate analyses for distant recurrence probability and, subsequently, for cancer-specific survival. Identifying stage I and II patients at risk for distant disease recurrence and assessing primary tumors for predictive genomic biomarkers would be important for stratifying adjuvant treatment. Moreover, given that accurate upstaging from stage II to III remains a difficult task, ${ }^{34}$ we approached this by a quantitative analysis of a specific panel of epigenetic biomarkers. The advantage of using genomic analysis is the stability of DNA compared with mRNA in PEAT, where, in the latter, there is a higher level of degradation with time. Additional studies will involve validation in a prospective clinical trial.

\section{AUTHORS' DISCLOSURES OF POTENTIAL CONFLICTS OF INTEREST}

The author(s) indicated no potential conflicts of interest.

\section{AUTHOR CONTRIBUTIONS}

Conception and design: Michiel F.G. de Maat, Cornelis J.H. van de Velde, Martijn P.J. van der Werff, Naoyuki Umetani, Elma Meershoek 
Klein-Kranenbarg, J. Han J.M. van Krieken, Rob A.E.M. Tollenaar, Dave S.B. Hoon

Financial support: Cornelis J.H. van de Velde, Dave S.B. Hoon Administrative support: Cornelis J.H. van de Velde, Dave S.B. Hoon Provision of study materials or patients: Cornelis J.H. van de Velde, Roderick R. Turner, Anton Bilchik

Collection and assembly of data: Michiel F.G. de Maat,

Roderick R. Turner

Data analysis and interpretation: Michiel F.G. de Maat, Cornelis J.H. van de Velde, Martijn P.J. van der Werff, Hein Putter, Naoyuki Umetani,
Elma Meershoek Klein-Kranenbarg, J. Han J.M. van Krieken, Rob A.E.M. Tollenaar

Manuscript writing: Michiel F.G. de Maat, Cornelis J.H. van de Velde, Martijn P.J. van der Werff, Hein Putter, Naoyuki Umetani, Elma Meershoek Klein-Kranenbarg, Roderick R. Turner, J. Han J.M. van Krieken, Rob A.E.M. Tollenaar, Dave S.B. Hoon Final approval of manuscript: Michiel F.G. de Maat, Cornelis J.H. van de Velde, Martijn P.J. van der Werff, Hein Putter, Naoyuki Umetani, Elma Meershoek Klein-Kranenbarg, Roderick R. Turner, J. Han J.M. van Krieken, Rob A.E.M. Tollenaar, Dave S.B. Hoon

\section{REFERENCES}

1. Jemal $A$, Siegel $R$, Ward $E$, et al: Cancer statistics, 2007. CA Cancer J Clin 57:43-66, 2007

2. Improved survival with preoperative radiotherapy in resectable rectal cancer: Swedish Rectal Cancer Trial. N Engl J Med 336:980-987, 1997

3. Kapiteijn E, Marijnen CA, Nagtegaal ID, et al: Preoperative radiotherapy combined with total mesorectal excision for resectable rectal cancer. N Engl J Med 345:638-646, 2001

4. Bosset JF, Collette L, Calais G, et al: Chemotherapy with preoperative radiotherapy in rectal cancer. N Engl J Med 355:1114-1123, 2006

5. lacopetta B: Are there two sides to colorectal cancer? Int J Cancer 101:403-408, 2002

6. Jones PA, Gonzalgo ML: Altered DNA methylation and genome instability: A new pathway to cancer? Proc Natl Acad Sci U S A 94:2103-2105, 1997

7. Jones PA, Baylin SB: The fundamental role of epigenetic events in cancer. Nat Rev Genet 3:415428, 2002

8. Baylin SB, Herman JG: DNA hypermethylation in tumorigenesis: Epigenetics joins genetics. Trends Genet 16:168-174, 2000

9. Zeschnigk $M$, Bohringer S, Price EA, et al: A novel real-time PCR assay for quantitative analysis of methylated alleles (QAMA): Analysis of the retinoblastoma locus. Nucleic Acids Res 32:e125, 2004

10. de Maat MF, Umetani $N$, Sunami $E$, et al: Assessment of methylation events during colorectal tumor progression by absolute quantitative analysis of methylated alleles. Mol Cancer Res 5:461-471, 2007

11. Umetani $N$, de Maat MF, Sunami $E$, et al: Methylation of p16 and Ras association domain family protein 1a during colorectal malignant transformation. Mol Cancer Res 4:303-309, 2006

12. Toyota $\mathrm{M}, \mathrm{Ho} \mathrm{C}$, Ahuja $\mathrm{N}$, et al: Identification of differentially methylated sequences in colorecta cancer by methylated $\mathrm{CpG}$ island amplification. Cancer Res 59:2307-2312, 1999
13. Issa JP, Shen L, Toyota M: CIMP, at last. Gastroenterology 129:1121-1124, 2005

14. Toyota M, Ahuja N, Ohe-Toyota M, et al: CpG island methylator phenotype in colorectal cancer. Proc Natl Acad Sci U S A 96:8681-8686, 1999

15. Toyota $\mathrm{M}$, Ohe-Toyota $\mathrm{M}$, Ahuja $\mathrm{N}$, et al: Distinct genetic profiles in colorectal tumors with or without the $\mathrm{CpG}$ island methylator phenotype. Proc Natl Acad Sci U S A 97:710-715, 2000

16. Toyota M, Ahuja N, Suzuki H, et al: Aberrant methylation in gastric cancer associated with the CpG island methylator phenotype. Cancer Res 59: 5438-5442, 1999

17. Wynter CV, Kambara T, Walsh MD, et al: DNA methylation patterns in adenomas from FAP, multiple adenoma and sporadic colorectal carcinoma patients. Int J Cancer 118:907-915, 2006

18. Peeters KC, Kapiteijn E, van de Velde CJ: Managing rectal cancer: The Dutch experience. Colorectal Dis 5:423-426, 2003

19. Kapiteijn E, van de Velde CJ: Developments and quality assurance in rectal cancer surgery. Eur $\mathrm{J}$ Cancer 38:919-936, 2002

20. Spugnardi M, Tommasi S, Dammann R, et al: Epigenetic inactivation of RAS association domain family protein 1 (RASSF1A) in malignant cutaneous melanoma. Cancer Res 63:1639-1643, 2003

21. Herman JG, Graff JR, Myohanen S, et al: Methylation-specific PCR: A novel PCR assay for methylation status of $\mathrm{CpG}$ islands. Proc Natl Acad Sci U S A 93:9821-9826, 1996

22. Umetani $N$, de Maat MF, Mori T, et al: Synthesis of universal unmethylated control DNA by nested whole genome amplification with phi29 DNA polymerase. Biochem Biophys Res Commun 329: 219-223, 2005

23. Liaw $A$, Wiener M: Classification and regression by random forest. $R$ News 2/3:18-22, 2002

24. Seligson DB, Horvath $S$, Shi T, et al: Global histone modification patterns predict risk of prostate cancer recurrence. Nature 435:1262-1266, 2005
25. Shi T, Seligson D, Belldegrun AS, et al: Tumor classification by tissue microarray profiling: Random forest clustering applied to renal cell carcinoma. Mod Pathol 18:547-557, 2005

26. Kapiteijn E, Kranenbarg EK, Steup WH, et al: Total mesorectal excision (TME) with or without preoperative radiotherapy in the treatment of primary rectal cancer: Prospective randomised trial with standard operative and histopathological techniques—Dutch ColoRectal Cancer Group. Eur J Surg 165:410-420, 1999

27. van Rijnsoever M, Grieu F, Elsaleh $H$, et al: Characterisation of colorectal cancers showing hypermethylation at multiple $\mathrm{CpG}$ islands. Gut 51:797802, 2002

28. Willingham AT, Gingeras TR: TUF love for “junk" DNA. Cell 125:1215-1220, 2006

29. Wang J, Gonzalez KD, Scaringe WA, et al: Evidence for mutation showers. Proc Natl Acad Sci U S A 104:8403-8408, 2007

30. Lin SL, Ying SY: Gene silencing in vitro and in vivo using intronic microRNAs. Methods Mol Biol 342:295-312, 2006

31. Weisenberger DJ, Siegmund KD, Campan $M$, et al: $C p G$ island methylator phenotype underlies sporadic microsatellite instability and is tightly associated with BRAF mutation in colorectal cancer. Nat Genet 38:787-793, 2006

32. Ward RL, Cheong K, Ku SL, et al: Adverse prognostic effect of methylation in colorectal cancer is reversed by microsatellite instability. $\mathrm{J}$ Clin Oncol 21:3729-3736, 2003

33. Hawkins N, Norrie M, Cheong K, et al: CpG island methylation in sporadic colorectal cancers and its relationship to microsatellite instability. Gastroenterology 122:1376-1387, 2002

34. Bilchik AJ, Nora DT, Sobin LH, et al: Effect of lymphatic mapping on the new tumor-nodemetastasis classification for colorectal cancer. J Clin Oncol 21:668-672, 2003

\section{Appendix}

The Appendix is included in the full-text version of this article, available online at www.jco.org. It is not included in the PDF version (via Adobe ${ }^{\circledR} \operatorname{Reader}^{\circledR}$ ). 